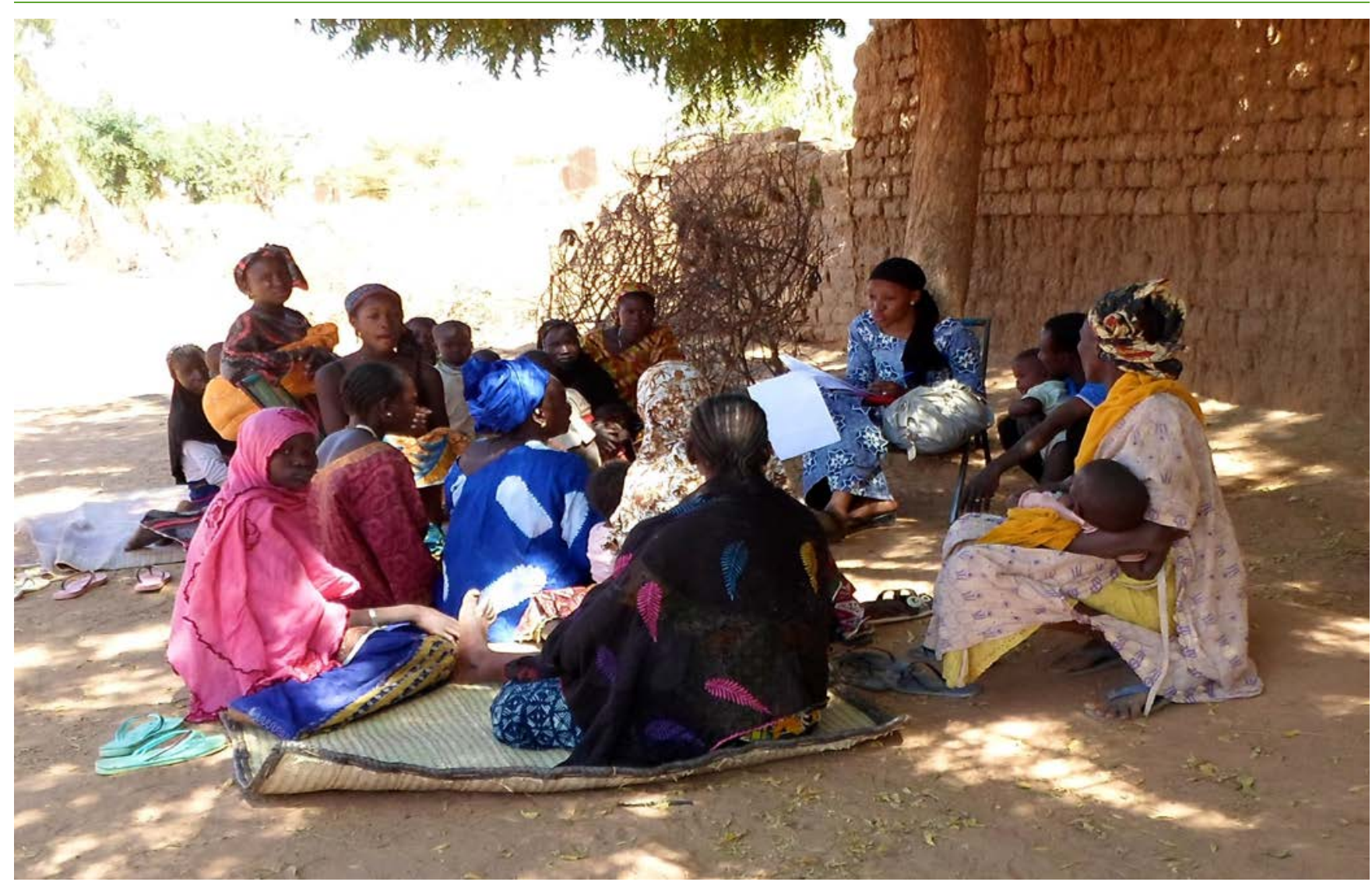

Focus Group in Douentza, Mali. Photo: Habibatou Gologo

\title{
COMMUNITY ENGAGEMENT IN SANITATION: A LANDSCAPE REVIEW
}

In 2017, Elrha's Humanitarian Innovation Fund (HIF) launched a Challenge 'to understand how to design, implement, and evaluate approaches to user-centred sanitation that incorporate rapid community engagement and are appropriate for the first stage of rapid-onset emergencies' (defined as the first twelve weeks post crisis). A component of this Challenge involved undertaking a landscape review of existing community engagement practice and approaches that could be used to provide a background resource for Challenge participants. The review was carried out by Oxfam, the HIF's Research and Evaluation Partner for the project. It draws on published and grey literature and interviews with 15 key informants. 


\section{CONTENTS}

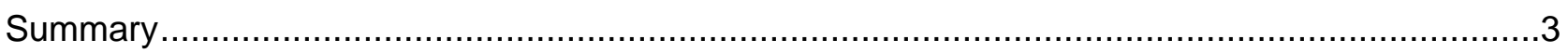

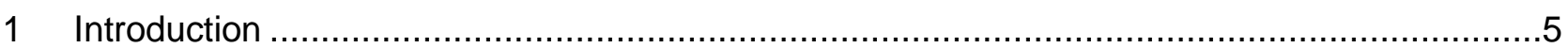

1.1 Why is community engagement in sanitation important? .............................................

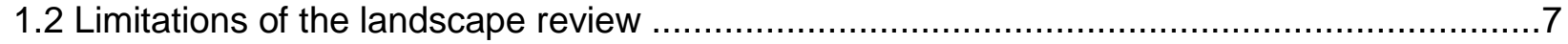

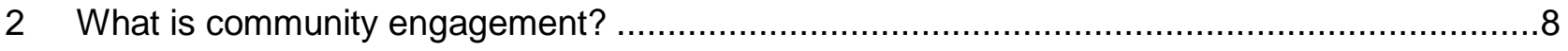

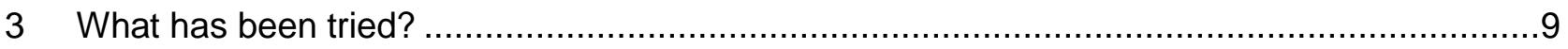

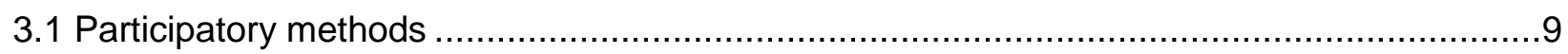

3.2 Participatory hygiene and sanitation transformation (PHAST) ................................ 10

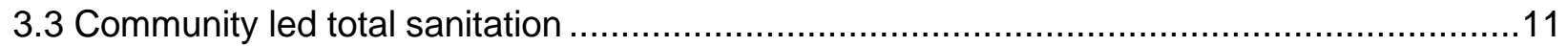

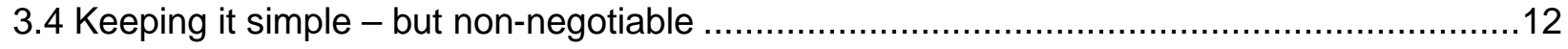

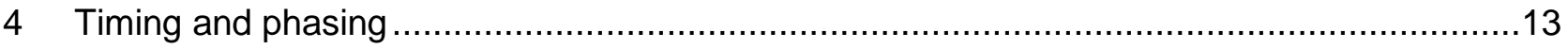

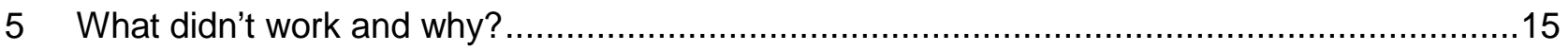

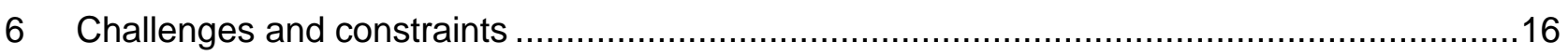

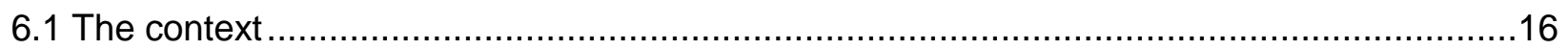

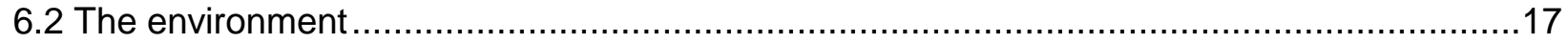

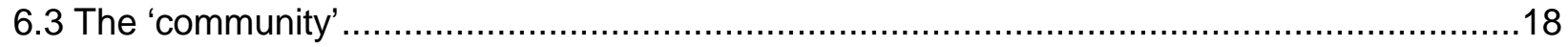

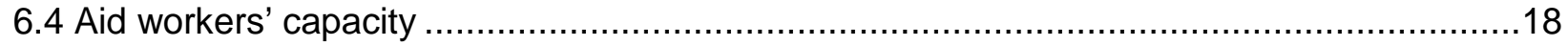

7 Monitoring \& evaluation of community engagement in sanitation...................................20

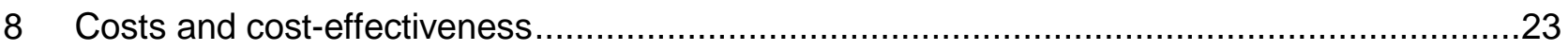

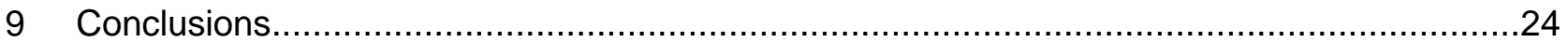

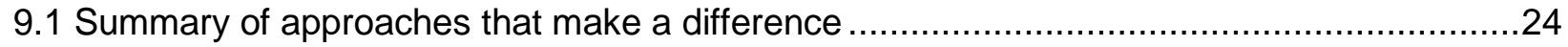

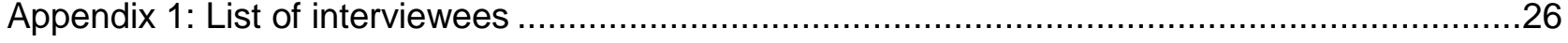

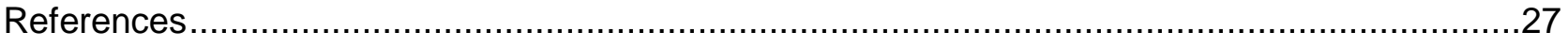

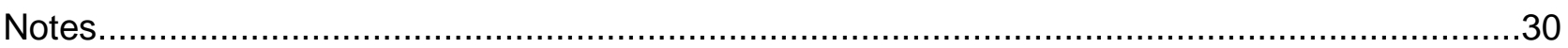




\section{EXECUTIVE SUMMARY}

Despite its perceived importance, the evidence suggests that community engagement in all sectors of humanitarian response is often limited and rarely monitored or evaluated. Sanitation projects may involve the community only in the construction phase as a paid labour force, or as a cashfor-work initiative.

One challenge for tracking the use and effectiveness of community engagement is that its meaning varies across the sector. Critically, agencies do not always define the aim of community engagement in their responses, even though it fundamentally affects how and what they do. Documented methods of community engagement in sanitation typically refer to standard participatory approaches, but were either used later in a response or did not specify the timing at all. Common community-based methods, adapted for WASH, include focus group discussions, community mapping, transect walks and key informant interviews. Whilst interviewees were positive about the benefits of community engagement, most commented that, in the first few weeks, it involved little more than a few conversations with groups of women and men, with people found at churches, mosques and informal gatherings, and with key informants from the community. Interviews with key informants for this review often stated that some level of engagement is always possible, despite the pressure to act fast. Even a few hours of basic consultation, done well, using respectful, open questioning with a limited (but representative) slice of the population, can positively influence sanitation design, community acceptance and ownership.

Several interviewees commented that approaches have to be simple; aid workers are often left to devise their own shortened versions of lengthy agency guidelines. Sometimes, uncertainty about what to prioritize and whether shortened approaches to engagement would prove effective leads to no engagement at all. Significant contextual constraints may override engagement (such as latrine construction being outsourced to contractors, lack of leadership and a supply-driven culture). Latrine design based on community preferences may not be feasible due to environmental factors (poor soils, concreted land). These challenges combine with the complexity and diversity of communities themselves and widespread uncertainty among aid workers about the skills required and the feasibility of engaging with communities when time is short and the risks are high.

Human attitudes to sanitation and to excreta are complex and culturally varied. Initial design, made in often chaotic and overwhelming circumstances, is just the beginning. It is difficult, and unlikely, that aid agencies can get it right first time. A key element of success appears to be phasing: to start with basic community engagement (e.g., consultation) and then develop and adapt over time. The challenge is that aid agencies appear to find it difficult to adopt a phased approach; practitioners lack guidance.

The limited availability of systematic and documented monitoring and evaluation of community engagement and sanitation also undermines learning. There is little for practitioners to draw upon for rapid-onset emergencies and work needs to be done to create quick and simple methods to measure the extent to which communities engaged with projects and with what effect. Proxy outcome indicators may be the most feasible and have been used successfully, such as monitoring levels of actual use of the sanitation facilities. 
Several interviewees suggested that access to a simple list of the critical information that must be sought from affected communities would help. Suggested elements (in no order of priority) included:

- the sanitation and anal cleansing materials that the affected community is accustomed to;

- gender considerations such as who normally shares a latrine (i.e. segregated by gender or by household);

- key cultural beliefs and attitudes to sanitation;

- protection considerations such as attitudes to privacy and safety and when people typically use latrines (day and night?), how far they will walk to visit a latrine;

- age-friendly considerations; and,

- the communities' capacity and role in maintaining and cleaning the sanitation facilities.

It is essential that such information is quickly fed into the sanitation design. This is often a weakness of project design, since engineers may not themselves engage with the community, increasing the risk that community views do not influence sanitation design. Several interviewees suggested that community engagement should always involve some engineers at least some of the time, even if the process is led by those with community-based expertise. Designs will usually need to be revisited and redesigned over time. Projects should plan for iterations and recognize when the context permits fuller engagement: start simply and adapt and deepen later.

There needs to be a courageous redefinition of what community engagement can actually entail, and for what purpose, in the first few weeks of a rapid-onset emergency. Genuinely minimum standards or principles for guiding engagement are needed that are simple and portable - aiming for a sufficiency that is still effective and can be adapted to different contexts. The creation of guidelines of no more than a page could be a brave and appropriate tool in the first weeks of an emergency.

If the HIF Challenge projects can capture, through well-documented monitoring and evaluation, approaches to community engagement that are quick and feasible in rapid-onset emergencies, leading to appropriate and effective sanitation, it would be a major innovation, even if the methods themselves are nothing new. 


\section{INTRODUCTION}

In 2017, the Humanitarian Innovation Fund (HIF) launched a Challenge 'to understand how to design, implement, and evaluate approaches to user-centred sanitation that incorporate rapid community engagement and are appropriate for the first stage of rapid-onset emergencies' (HIF, 2017). A component of the HIF Challenge was to carry out a landscape review of existing community engagement practice and relevant approaches that could be applied in rapid-onset emergencies. The review has been carried out by Oxfam, the HIF's Research and Evaluation Partner for the project, and the findings presented in this paper.

The HIF Handbook defines user-centred as when the 'design tries to optimize the product (or service) around how users can, want, or need to use the product (or service), rather than forcing the users to change their behaviour to accommodate the product (or service)'. Community engagement (in sanitation) is defined as 'Actions and processes taken to understand the basic needs, preferences and cultural practices of emergency-affected communities in order to design and implement relevant and appropriate sanitation solutions'. Sanitation is defined as the 'provision of facilities and services for the safe disposal of human urine and faeces' and rapid as a 'sudden emergency with sanitation selected and implemented in less than 12 weeks of the start of a crisis' (HIF, 2017).

The review has been compiled through a literature search and semi-structured interviews with 15 key informants in the humanitarian and development sanitation field. Of the 40 documents reviewed, some 33 were relevant to this review. The literature was evenly split between published and grey literature. ${ }^{1}$ The main review questions were:

- what has been tried, in what contexts and what was successful or unsuccessful and why;

- which models of participation were used and how were they measured and monitored;

- did external factors such as sector standards, government and donor policies and particular contexts influence approaches and effectiveness;

- how were feedback mechanisms and technologies used to collect and analyse data; and

- how costly and cost-effective were community engagement approaches?

\subsection{WHY IS COMMUNITY ENGAGEMENT IN SANITATION IMPORTANT?}

Safe sanitation, along with clean water and basic hygiene measures, is known to be a critical intervention in emergencies, preventing or reducing disease and its transmission. According to the World Health Organization (WHO) website, 'Diarrhoeal disease alone amounts to an estimated 3.6\% of the total DALY global burden of disease and is responsible for the deaths of 1.5 million people every year' (WHO, 2012). It is estimated that $58 \%$ of that burden, or 842,000 deaths per year, is attributable to unsafe water supply, sanitation and hygiene. ${ }^{2}$

In 2017, 3iE carried out a systematic review of the effectiveness of short-term WASH interventions. It identified engaging with the affected communities as one of four key design considerations (Yates et al, 2017a). 
The importance of engaging with affected populations was recognized by the Sphere Minimum Standards as early as 2004. By the 2011 edition, the first Sphere WASH standard on programme design and implementation states that 'WASH needs of the affected population are met and users are involved in the design, management and maintenance of the facilities where appropriate'. Sphere's excreta disposal standard 2 ('Appropriate and adequate toilet facilities') promotes consultation and approval from users on the siting, design and appropriateness of sanitation facilities in order to allow rapid, safe and secure access.

Despite its perceived importance, the evidence suggests that community engagement in all sectors of humanitarian response is often limited. In its 2012 report State of the Humanitarian System, the Active Learning Network for Accountability and Performance (ALNAP) states that 'Overall it finds the weakest progress and performance in the areas of recipient consultation and engagement of local actors, despite the rhetorical emphasis given to these issues' (ALNAP, 2012). Engagement may be confined, at best, to basic consultation. Participation in sanitation projects may involve the community only in the construction phase as a paid labour force, or as a cash-for-work initiative.

Practitioners also lack the time or resources to record their experiences, so even if communities are engaged, the results are not widely shared. The impact of community engagement is rarely studied in humanitarian evaluations or research. One study did set out to link Humanitarian Accountability Partnership (HAP) benchmarks with outcomes for information sharing, participation and complaints handling. The 'findings of the research provide significant evidence of the link between accountability mechanisms and the relevance, effectiveness, efficiency and sustainability of projects' (Featherstone, 2013). The report states that as well as generating trust and increasing ownership, 'Community participation provided agencies with a better understanding of local vulnerabilities and increased the usefulness of projects to communities'.

Drawing on evaluation evidence, the 2017 3iE WASH review notes that 'Overall, social mobilization was effective at reducing disease risk, output of structures and building stronger community relationships' (Yates et al, 2017a). The findings suggested that 'Engaging with specific populations was not burdensome but led to more appropriate latrine designs (e.g. locking doors, handrails) with marginal additional costs. In South Sudan, female use of latrines was significantly higher $(p<0.001)$ where this gender targeting was done compared to another camp in the area (de Lange et al. 2014)'. Interestingly, the report notes that 'While each context is unique, it was consistently found that beneficiaries will use latrines provided they are safe, clean and offer privacy.' The same study also notes that the quality of the evidence is often poor and that overall, evidence of the effectiveness of community engagement is scarce. Scarcer still is data about participation in sanitation projects.

The impact of WASH interventions (and sanitation in particular) on health outcomes itself lacks an evidence base. The 3iE study found that 'numerous methodological limitations limit the ability to determine associative, let alone causal, relationships. Of those studies considered to be of sufficient relevance and quality, only 4 percent addressed sanitation' (Yates et al., 2017a). Another extensive study searched for documented evidence of the health impact of sanitation on cholera. Out of 1,291 documents identified in the initial search, none were specifically on sanitation (Cairncross et al., 2009). The report notes that 'Further research is necessary in order to identify techniques that can promote usage of latrines for instance integrating the community within the design, construction and maintenance of latrines in order to create a sense of ownership'.

Although finding impact evidence on health outcomes is not the aim of the HIF project, the wider lack of sanitation data partly explains the current gaps in knowledge about engaging communities within the sanitation field. 
In 2013, a HIF-funded gap analysis in emergency WASH identified sanitation issues 'as the major area with gaps and potential for innovation' (Bastable and Russell, 2013) and 'The other major issue highlighted was weak community participation and the critical importance of designing appropriate hygiene promotion activities for each context'. These weaknesses have led to the current HIF Challenge, which aims to help fill gaps in documented knowledge by supporting and testing innovative approaches to rapid community engagement in sanitation, monitoring the methods used and recording their effect on the design, provision, appropriateness and use of facilities.

\subsection{LIMITATIONS OF THE LANDSCAPE REVIEW}

Searches for documented approaches and learning from community engagement in rapid sanitation responses yielded no relevant direct results. Wider searches, incorporating search terms covering humanitarian, post-emergency, recovery, rehabilitation, WASH more broadly and non-WASH interventions yielded a limited number of relevant documents. The most frequently documented approaches were based on Community Led Total Sanitation (CLTS) in post-emergency phases and in fragile and conflict affected states. A case study was also identified using Participatory Hygiene and Sanitation Transformation (PHAST).

As a result of the limited documented evidence, the landscape review search was expanded to include feedback obtained through semi-structured interviews with 15 key informants. The interviews yielded invaluable additional material and internal reports, although little detail was available on methods or monitoring and evaluation. 


\section{WHAT IS COMMUNITY ENGAGEMENT?}

One challenge for tracking the use and effectiveness of community engagement is that its meaning varies across the sector. It refers to a number of ways in which aid workers and communities relate, communicate and implement a response. Methods of engaging with people include basic consultation with key informants, focus group discussions (FGD), information provision and feedback, transect walks, two- or three-pile sorting, community mapping and physical engagement (e.g. provision of labour or materials). Community engagement can also refer to accountability approaches such as information sharing and complaints mechanisms. Community engagement often describes an overall programme approach which aims to share power with affected people through their active influence and participation in design, decisions about implementation, and monitoring and evaluation. According to the HIF Handbook for this Challenge, community engagement 'can range from observing users' behaviour to consulting with them on key decisions, or the co-design of sanitation facilities with communities' (HIF, 2017).

The purpose or intended effect of community engagement also varies. It includes a range of outcomes, from ensuring that community needs and preferences are understood (and then acted upon, leading to more appropriate interventions) to a wider commitment to accountability and to maximizing sustainability through enhanced community ownership. The process of engagement is often linked to empowerment objectives which aim to strengthen the voice and capacity of individuals and communities, challenging underlying social and political inequities. A paper commissioned by ALNAP (Brown et al., 2014) describes three main rationales for community engagement:

- 'normative' or value based (it is a human right and morally the right thing to do);

- 'instrumental' (the project will be better informed and more effective); and

- 'emancipatory' (addressing inequality and enhancing society).

The HIF Handbook describes the purpose of community engagement in largely instrumental terms: that 'it is crucial to engage with the affected communities to understand how to build sanitation they want and can safely access' (HIF, 2017).

Critically, agencies do not always define the aim of community engagement in their responses, even though it fundamentally affects how and what they do. Project approaches will, or should, differ depending on whether the hoped-for result is obtaining quality information or facilitating empowerment. If the intended outcome of community engagement is unclear, its effectiveness is hard to measure. 


\section{WHAT HAS BEEN TRIED?}

3.1 PARTICIPATORY METHODS

Documented examples of community engagement in sanitation at all phases in an emergency typically refer to standard participatory methods. They are adapted to WASH (or to sanitation in particular), but use common community-based methods such as FGDs, community mapping, transect walks and key informant interviews. An example of adapting standard tools to sanitation includes the use of community mapping in Nepal (Oxfam, 2015) to map out areas of open defecation, key risks and sanitation facilities.

Other community engagement methods mentioned in the documents and the interviews include identifying community leaders who are willing and able to represent the whole community, including camp management committees, public health community mobilizers, community volunteers and peer educators, as well as mothers' and children's clubs. The time taken to establish such clubs and committees is not typically recalled, and they may not be feasible mechanisms in the first few weeks of a response.

Overall, FGDs and key informant interviews were the methods most frequently referred to in the documents reviewed and interviews conducted for this review.

\section{Box 1: Oxfam Tanzania: Methods for engaging with children in sanitation in emergencies}

Oxfam's work with Burundian refugees in Tanzania used a number of participatory techniques to engage children. These included:

- Focus group discussions, separated by gender;

- A Yes/No game for children (used to find out what they know about sanitation and what they think about facilities). Yes/No or Don't Know cards are shown and children run to the relevant card when asked a question. This method can also be used for monitoring;

- Pocket chart voting (photos of different sanitation options are put up, with pockets below each, disaggregated by age group. People privately put a token in the chosen pockets to vote);

- Children's drawing exercises (e.g. draw the perfect toilet);

- Transect/observation walks;

- Community mapping (mapping out the location of latrines, hand-washing facilities etc. and discussing them);

- Ranking exercises - participants stand along a line marked 0-10 to rank how much they like the facilities can be used for different criteria such as design, usage, community involvement, cleanliness;

- Voting for different child-friendly latrines - facilitator demonstrates different options and asks for votes.

The exercises were engaging and fun for the children (and adults). The information led to smaller sanitation non-food items and child-friendly toilets. The exercises did not take long. Oxfam notes that although this exercise was conducted after the initial emergency trench latrines had been constructed, the team realize they could use the approaches much earlier, such as for adapting the design of rapid-onset communal latrines.

Several of the exercises can also be appropriate for adults.

CARE used its rapid gender analysis tool during the Nepal earthquake that happened 25 April 2015, collecting primary data through FGDs and key informant interviews over a period of two days in Sindhupalchowk. The analysis identified highly localized cultural gender issues. This led to design changes such as lighting and door locks, and 'a hook in the latrine so that girls and women can hang their purse and not be forced to place it on the floor. This dramatically improves use by women' (CARE, 2015). 
The approach was also used in South Sudan, where the findings led to alterations to the design of latrine security, segregation and type. The findings apparently influenced the WASH Cluster and the design of other agencies' latrines. However, although rapid, these gender analyses are typically undertaken a couple of months after the emergency's onset, leading to remedial action on existing facilities.

Save the Children emphasizes the importance of asking children key questions, which can be done rapidly (for example, to understand their fears, whether they are afraid of latrine holes or of being locked inside a latrine). As children can constitute 50-70 percent of a displaced population and their faeces are high risk, adapting designs to enable children to use latrines is critical. Save the Children use an adapted child-friendly latrine design (which also covers disabled needs).

There is also an increase in the use of digital data capture for community consultation, assessments and monitoring. Several agencies use KoBo, (field data collection software http://www.kobotoolbox.org), and staff are increasingly using tablets to record and, importantly, share information. Whether this enables the rapid exchange of information between the community and the engineers who will design the sanitation is not yet evident.

\subsection{PARTICIPATORY HYGIENE AND SANITATION TRANSFORMATION (PHAST)}

The International Federation of Red Cross and Red Crescent Societies (IFRC) has adapted PHAST for emergencies. 'When PHAST needs to be FAST' is a shortened version of the methodology. The Uganda Red Cross successfully used FAST PHAST in a cholera epidemic, aiming to find out "whether PHAST could successfully be shortened in [an] emergency without losing its impact and the community "buy-in" seen to be so essential to its successful implementation' (Gonzalez et al., 2006). The shortened PHAST uses three main methods: 3-pile sorting to find out about good and bad hygiene behaviour, the pocket chart tool for community sanitation and hygiene practices, and tools to explore transmission routes and blocking disease routes. Other PHAST tools (such as planning tools and discussion) were left until later. The activities were accompanied by an intensive house-to-house hygiene messaging campaign. IFRC found that with PHAST, 'National Societies that have been working on participatory methodologies as part of the emergency prevention and preparedness are able to introduce the participative elements in the response efficiently since the very beginning' (ibid., p. 1).

IFRC also uses the 'HP Box', a ready-to-go kit which includes hygiene promotion materials (such as banners, stationery, puppet shows). According to the IFRC, this has had a significant effect on staff capacity in rapid onset emergencies, as the availability of the HP Box increases staff capacity to initiate early participatory techniques, irrespective of the local availability of appropriate materials and their previous experience.

As with other forms of hygiene promotion, PHAST's objectives are concerned with hygiene behaviour change through transferring knowledge about disease transmission and hygiene behaviours to the affected communities. Arguably, hygiene promotion differs from community engagement because, while it still requires engagement, the objective is more didactic. Behaviour change (such as using latrines) may also increase through a better awareness of a community's motivation, which isn't necessarily to reduce health risks. 'Research has shown that factors such as emotional drivers, social status or affiliation, disgust or aesthetics are more likely to provide the motivation for change than health-based messages' (Ramos et al., 2016). Save the Children's rapid sanitation response in the Philippines was not only about health messaging but also about 'making latrines cool' (Dyah and Chang, 2014). The value and sense of 
ownership of a latrine was strongly linked to their value as a 'must-have' item and its associated social standing. This and other examples suggest the potential for community-driven sanitation in emergencies, particularly in host communities and other non-camp contexts. However, as the actual timing of the process is not known, it is not possible to assess its feasibility in rapid-onset.

The majority of the humanitarian community engagement examples found for this study aimed to enhance the appropriateness of the facilities and to encourage ownership (often linked to maintenance and cleaning needs). Longer term objectives such as sustainable sanitation or empowerment were rare and mostly found in CLTS approaches.

\subsection{COMMUNITY LED TOTAL SANITATION}

\section{Box 2: Community Led Total Sanitation (CLTS) and adaptations of it, such as Community Approaches for Total Sanitation (CATS)}

Such approaches place a strong emphasis on long-term, sustained access to sanitation. Projects are initiated only if sanitation is demanded by the community. The emphasis is on the entire community rather than single households. Community 'natural leaders' play a significant role, one which can be effectively adopted by children. There is a considerable amount of written information about the effectiveness of CLTS. An example from Liberia is cited in the 3iE review, where "households in villages that achieved "open defecation free" through CLTS were 17 times less likely to have cases of Ebola than non-CLTS communities'. Its success in terms of longer term positive sanitation behaviours has led to it being adopted by several governments, as well as UNICEF.

However, CLTS has largely been used in development, post-emergency and recovery contexts. A UNICEF study warns that 'focusing on disgust might not be appropriate/ethical to people who have already experienced shock' (Greaves, 2016). Although CLTS can be carried out quickly, in an emergency it is not ethical to walk away from a community that has not been 'triggered' ('triggering' is based on stimulating a collective sense of disgust and shame among community members). Moreover, displaced communities with weak social cohesion, the presence of multiple supply-driven responses and, often, a total lack of community resources, make CLTS appear inappropriate for rapid-onset sanitation. The literature does leave the question open as to whether adapted versions - in particular those that provide some incentives or help, such as the loan of latrine digging tools - can make CLTS appropriate far earlier in emergencies than thought. It has been successfully used in camp situations (e.g. in Pakistan), despite low social cohesion. CARE in Pak Sindh found CLTS to be effective in bringing about behaviour change in people who were unaccustomed to using latrines and had been using the latrine superstructures as a privacy screen for open defecation. Tearfund provided prefabricated (lightweight) slabs in Iraq, realizing that the communities were likely to move on and needed the assurance that their investment in the sanitation could be taken with them. In Nepal, Tearfund negotiated a 'Kick-Start ODF' adaptation to CLTS with the government, providing a minimum package of latrine-building materials for the vulnerable people (Greaves, 2016).

As Greaves (2016) notes, 'as time progresses, priorities and human capacities change, sanitation itself will become a key consideration for health and well-being, and conditions for CLTS self action often improve.' The question remains open as to how early CLTS could be used and whether it can or should be included in the menu of approaches used by aid agencies, should the context permit. 


\subsection{KEEPING IT SIMPLE - BUT NON-NEGOTIABLE}

Several interviewees commented that approaches have to be simple; aid workers are often left to devise their own shortened versions of 'castles in the air' - lengthy agency guidelines that have little realistic chance of being implemented (or perhaps, even read) in rapid-onset emergencies. Sometimes, the uncertainty about what to prioritize and whether shortened approaches to engagement would be effective leads to no engagement at all. Some interviewees stressed that any guidance should be limited to one page - increasing the chances of it being read and applied. An expert on hand-washing research observed that understanding the drivers for behaviour change is complex and takes time and that it is important to identify earlier opportunities for changing behaviour without an in-depth understanding, as a temporary holding position. One such approach is that of 'nudges' - for example, painted footprints on the ground leading to sanitation facilities.

As well as being simple, the ambition of initial community engagement should be modest - but nonnegotiable. Interviews with key informants for this review often stated that some level of engagement is always possible, despite the pressure to act fast. The immediate priority is to gather feedback from affected people about important basics such as the type of sanitation they are used to, attitudes to privacy and strongly established cultural preferences and taboos. Interviewees made a number of suggestions about what affected people must be consulted about, at minimum. These are collated below in Box 3 (in random order).

\section{Box 3: Minimum topics to be covered in a consultation process}

- What sanitation structures the affected community are accustomed to (e.g. pour flush, direct drop, open defecation and the materials used for anal cleansing);

- Gender considerations such as who normally shares a latrine (i.e. segregated by gender or by household); widely displaced people and refugees may not be from the same area or ethnic and religious background: will it be acceptable for them to share with other groups?

- Protection considerations including cultural beliefs and attitudes to sanitation (what is taboo, what is safe and dignified);

- Attitudes to privacy and safety;

- Age-friendly considerations;

- When people typically use latrines (day or night? How far will they walk to visit a latrine at night, and does this vary according to sex and age); and

- the communities' capacity and role in maintaining and cleaning the sanitation facilities. 
Rapid obtainment of information is essential, so a good rule is to 'gather fast, talk more later'. The risk is that deeper engagement with communities never develops beyond this. Yet interviews suggested that initial, albeit limited, consultation creates the foundation for greater engagement later on. It is the beginning of a process, not a single opportunity. Some of the engineers interviewed described a typical phasing of sanitation as follows:

1. Phase I - Emergency communal trench latrines using squatting slabs and plastic sheeting superstructures

2. Phase II - Stabilized and improved trench latrines with rigid emergency superstructures

3. Phase III - Shared latrines - by families or groups. More durable superstructures

4. Phase IV - Depending on the context, household latrines with durable superstructures

Phase 1, according to interviews, is typically carried out with limited, or even no community engagement. The provision of communal latrines reduces the immediate risks and provides a 'breathing space' to permit further engagement. In some contexts, allowing open defecation (OD) but making it safe (e.g., by demarcating OD areas that will not contaminate the water or wider environment) has proved to be the most appropriate strategy when communities are neither accustomed nor motivated to use latrines, at least in the first phase. The initial consultation may involve little more than a few conversations with groups of women, with people who were approached at churches, mosques and informal gatherings, and with key informants from the community. Done well, with respectful, open questioning with a limited but representative slice of the population, this can still positively influence sanitation design. Once the priority of immediate, safe management of excreta is achieved, further engagement (and ongoing feedback on the existing facilities) refines the design.

Practitioners observed that a phased approach is not a linear process. The reality is much messier, and different phases can begin at the same time. For example, the digging of the trenches for latrines can begin whilst consultation about the superstructure is taking place. At the same time that communal sanitation is being improved, further engagement with people can begin regarding the design of family latrines or other forms of sharing by smaller groups. The shift from communal to family/shared latrines is a significant opportunity: the establishment of better facilities, such as bathing areas, requires deeper engagement between aid agencies and communities. Critical facilities such as menstrual hygiene management and community involvement in ongoing operation and maintenance (O\&M) can be better addressed. One interviewee pointed out that initial communal trench latrines do not become redundant when the design switches to family latrines - the same trenches can be used. It is the superstructure which changes.

Initial design, made in often chaotic and overwhelming circumstances, is just the beginning. Several interviewees commented that apparently simple design features, such as ensuring that latrine doors can be 'locked' for privacy and safety, can prove challenging in the early phase of an emergency (rapidly constructed superstructures made of plastic sheeting do not permit locks). Engineers may innovate (a nail and string), but solutions are not always immediate or obvious. Solutions for some of the community (who prefer locked doors) may turn out not to suit others (children may be afraid of being locked in). The International Rescue Committee (IRC), which had consulted women in camps in Iraq, had understood their preference to be for household latrines. Following subsequent engagement and the development of trust, aid workers discovered that the women didn't actually like shared household latrines after all, preferring them to be separated by sex, not household. Oxfam in Pakistan realized that it was culturally unacceptable for women to be seen going to the toilet, so they joined the toilets with shower units to enable privacy. 
None of these adaptations took place in the first phase but formed part of the evolution of the project.

Human attitudes to sanitation and to excreta are complex and culturally varied. In some parts of South Sudan, for example, faeces are associated with power. It is therefore unacceptable to defecate immediately on top of the faeces of someone higher up the hierarchy. In some cultures, women may prefer to share toilets with other women, rather than with men in their own family. It is difficult, and unlikely, that aid agencies can get it right first time. Early designs may also have unexpected and unintended consequences. In South Sudan, Oxfam provided lighting in latrine locations to improve safety for women visiting the toilets at night. The lighting was also attractive to young men (often returning fighters), who congregated in the newly lit areas to drink and play cards; women subsequently stopped using the toilets. This doesn't represent a mistake in community consultation; rather, it demonstrates that changes can have unpredictable results. A key element of success appears to be adapting over time.

The challenge is that aid agencies find it difficult to adopt a phased approach. This can be due to the attitude or mandate of a particular agency or team (some agencies aim exclusively for first-phase coverage with communal latrines, typically designed without consultation, after which they leave).

The implementation of a rapid response project is accompanied by funded (and seemingly inflexible) proposals that struggle to incorporate iteration and alteration. Aid workers can get locked into a rapid response mindset and don't recognize when the context permits greater time investment in community engagement. Moreover, agency guidelines on community engagement and WASH tend not to offer advice about what to prioritize early in an emergency and how to develop and adapt community engagement over time. According to a survey of 20 Accord WASH agencies, whilst agencies are clear about what to do, their main challenge is 'knowing when to employ them in the midst of complex and dynamic environments' (Armstrong, 2016). The report suggests that greater attention is needed to contextual factors which mark changes in demand for WASH services 'as necessity transitions to convenience'.

'Where is a woman's dignity if she has to carry a bucket of water and walk between tents each time she wants to use the toilet?'

Syrian refugee, quoted in IRIN (2013) 


\section{WHAT DIDN'T WORK AND WHY?}

Most examples of poor practice describe an absence of community engagement, not weaknesses of particular community engagement and sanitation approaches.

A distressing and expensive example of a failure to consult people occurred in the Za'atari camp for Syrian refugees in Jordan. Latrines were widely vandalized as they did not meet fundamental cultural and safety needs. For the women, being seen in public visiting a latrine was neither safe nor culturally acceptable. The people took action: according to an IRIN report, UNICEF estimated that 'nearly one-third of refugees have built their own toilets next to their tents, the majority of them stealing metal sheets and cement blocks from the public bathrooms to do so. UNICEF estimates it has lost US\$1.5 million in damage to its water and sanitation facilities'. 'UNICEF now recognizes this kind of community mobilization is essential to engage the refugees, involve them in project development, and give them a sense of ownership in the process, making them less willing to destroy what they themselves have built' (IRIN, 2013).

Sanitation projects do not take place in isolation. Design must take account of whether other needs are being met (and sanitation may not be people's first priority). In Tanzania, communities were slashing and stealing plastic sheeting - because there was a gap in shelter provision. Hand-washing stations cannot succeed if there is inadequate provision of water for drinking and domestic use. Community preferences for water for latrine flushing cannot be incorporated if water supplies are limited. Not all community preferences may be technically feasible. 


\section{CHALLENGES AND CONSTRAINTS}

The main challenges identified in this study concern the context (government and donor policy and Cluster or other aid leadership), aid worker attitudes and skills, our notions of 'community' and the constraints of the environment. A real, and perceived, lack of time and resources cuts across many of these challenges.

\subsection{THE CONTEXT}

Early phase major emergencies often become highly supply driven; the pressure to demonstrate quantitative progress (e.g. high numbers of latrines built) can undermine attempts to ensure quality. In some contexts, latrine construction is outsourced to contractors who have little experience of, or incentive, to consult with people. Preparedness and risk reduction strategies meant that latrines in Northern Iraq were often built before the affected people had arrived. Many agencies, to ensure a rapid response, anticipate needs based on prior experience and order their sanitation kit before any conversations have been had. In these cases, secondary data about the affected communities is critical (though anecdotally not always referred to). The goal of community engagement must often focus on post-construction design adaptations and retrofitting.

The approach to sanitation of humanitarian agencies can undermine the efforts of another to encourage greater community participation and ownership. If an agency is constructing latrines with imported or expensive materials and providing payment to affected communities, then a participatory approach offering only local materials may appear to be an inferior and unwelcome option, even if the ownership and durability of the sanitation facilities would be enhanced. The evidence from more stable, developmental contexts suggests that successful and sustained safe sanitation results only from community owned and led initiatives. In emergencies, however, community-led sanitation may not be appropriate or feasible. The community is usually traumatized and may not constitute a cohesive group that is willing or able to work for a wider good, particularly in urban contexts. The context may also be highly volatile, with continual new arrivals and an expectation among all that they will return home soon, reducing the incentive to invest in temporary facilities.

Some agencies pay the community to carry out tasks (latrine cleaning, construction), arguing that communities early in an emergency do not prioritize sanitation and are unwilling to work for free. The question of payments and incentives was often raised in interviews as a significant challenge, since payments establish expectations, promote dependency and undermine community ownership. Some interviewees stated that the use of incentives (rather than payment), such as clothing or cleaning materials is an acceptable compromise. There have been cases where, if properly managed, with good relationships, acceptance and communication, people will accept increasing levels of responsibility over time. This is particularly so if the contested issue of payment is coherently dealt with by all actors and led by the WASH Cluster or government. 


\section{Urban sanitation}

Urban sanitation designers will typically face often complex land ownership and municipal regulations. Urban communities may be far less socially cohesive than small rural communities. Aid workers will typically have to engage with municipal authorities: those responsible for sanitation and for community initiatives may span multiple ministries, adding to the complexity and reducing the amount of time available for engaging with the affected people themselves. There are likely to be regulations in place as well as pressure from the authorities to upgrade existing urban WASH infrastructure, rather than focus on user-led solutions.

Government policy and WASH Cluster leadership can be both an enabler and a challenge. The government of Nepal re-established a CLTS approach four months after the 2015 earthquake, prompting agencies to shift to a user-led sanitation approach. The Ethiopian government similarly discouraged agencies from providing materials, re-establishing community-led sanitation. At the same time, if the policy shift is premature, affected people returning to a decimated environment with little or no resources are unable to provide local materials and contributions. Adapted CLTS approaches (see Box 2) have been tried, whereby materials are provided for vulnerable groups. It is too early to say whether such compromises have impacted negatively on sustained community ownership of sanitation. Cluster leadership of community engagement and minimum standards can be a significant enabler (for example, a Cluster may require agencies to ensure all latrines had lockable doors). One interviewee commented that the Cluster does not always recognize or use its authority to push for such minimum standards.

The terms of reference for this study included an assessment of whether the World Humanitarian Summit's Grand Bargain ${ }^{3}$ had led to any changes in community engagement in sanitation. No literature found referred to the Grand Bargain. Given the rapid-onset timeframe being reviewed here, the relevance of, for example, multi-year funding, is low. Funding that is not earmarked for specific purposes could be given to local providers and would, potentially, influence the ability of responders to fund community engagement, but there is no evidence as yet to draw upon.

Many of the examples used in this study are from camp contexts. Although engaging with host communities was referred to, no learning was found regarding differences in approach to community engagement in different contexts.

\subsection{THE ENVIRONMENT}

Practitioners commented that latrine options can be severely limited in many environments: soil stability, lack of space, lack of locally available materials, sloping land and limited access can all prevent sanitation designers from incorporating community preferences. Urban environments add to the challenges, as engineers may have to devise a 'no dig' solution in concreted areas. 


\subsection{THE 'COMMUNITY'}

The term community is often used to describe an apparently homogenous group with shared values, culture and needs. The reality may be considerably different. Communities are diverse (in ethnicity, wealth, age and sex, rural or urban, capacity, health and, particularly among refugees, in nationality). They have differing needs and levels of social stability. Disaster-affected people are also likely to be traumatized and exhausted and may not have the capacity or interest to engage. They may also be subject to numerous demands on their time from multiple agency interventions and be weary of 'participating'. Sanitation was often described as a relatively low priority among affected people early in an emergency: water, food and shelter command more attention. People have also been exposed to intense levels of change; some interviewees noted that they struggle to absorb new information and forms of engagement. One interview said that affected people can also be more receptive to change and new approaches.

No response or community is the same. The findings suggest that we should be careful regarding our assumptions about the affected 'community' and modest in our expectations of them. Some argue that the reality of community engagement is simply a means to an end, where the 'end' is latrines that are used, cleaned and maintained. 'The addition of positive and inclusive terms such as "engagement", "ownership" and "participation" jar with the realities of programmes which, in effect, roll out preformed plans on populations' (Wilkinson et al., 2017). An ALNAP paper asks whether we should 're-envision the humanitarian relationship as a contractual one rather than as an unequal exchange. In a contractual relationship, all sides know what to expect - what will be done in exchange for what - in a deal without sentimentality or rhetoric' (Brown et al., 2014). This would at least clarify expectations and demonstrate honesty and modesty. Either way, the findings suggest that aid workers should: avoid making assumptions about what form and level of engagement is appropriate; adapt to the particular context and people; and be clear and pragmatic about the hoped-for result of community engagement.

\subsection{AID WORKERS' CAPACITY}

Community engagement requires particular skills, for example, in facilitation. WASH personnel may lack these skills and the confidence to test them. Several interviewees noted that staff with pre-emergency training or experience were far more likely to integrate community engagement approaches into WASH response. As with all sectors, high turnover of personnel makes training and the widespread use of established guidelines challenging. Agency guidelines are often too lengthy to be practical in rapidonset emergencies and are rarely prioritized to the bare minimum that is often the reality for community engagement in the first few weeks of an emergency. As this report has found, there is little documented evidence of effective, rapid community engagement, further constraining learning.

The old caricature of the 'cowboy' engineer who is a 'know-it-all', ignoring non-technical information, was referred to by some interviewees, indicating that preconceived attitudes remain a barrier. Most, however, thought that weak or absent community engagement was caused mainly by lack of skills, leadership and time, all combined in a chaotic context where the pressure to produce tangible results is intense. WASH staff may have either engineering or community/hygiene promotion backgrounds. Some agencies have separate posts for engineers and community engagement experts. The latter are often given sole responsibility for engaging with communities, whilst the engineers proceed with construction. This can 
apparently greatly increase the risk that community consultation is not integrated into engineering designs, according to some of the interviewees.

Several interviewees suggested that community engagement should always involve engineers, even if the process is led by those with community-based expertise. This idea is supported by several comments referring to the role of Programme Managers, who have an essential role to play in the integration of engineering design and community engagement. However, in rapid-onset emergencies, they are frequently caught up in multiple coordination meetings and other internal or media communication duties, scarcely finding time to engage with field teams, programme design and quality. The presence of engineers/sanitation designers in community engagement also mitigates the risk that community workers will inadvertently raise expectations: engineers can quickly inform communities about the technical constraints, engaging them in the design of acceptable compromises. This is not to suggest that all engineers must engage with all community processes - this would exacerbate the workload of engineers and delay construction - a representative with the authority to convey community feedback to the rest of the engineering team will suffice.

Uncertainty about the feasibility and effectiveness of rapid community engagement doesn't help. Should aid workers delay the construction of latrines in order to talk, when the disease risk is so high, when surely a top-down approach is faster and saves more lives? What if rapid community engagement actually does more harm - by creating the illusion that the community is represented, when lack of time might mean only the leaders and the loudest are speaking for the community? Given technical or land constraints, is there any point in engaging with the community when it might raise expectations? How should aid workers balance the flow of expertise between engineers (who know what is technically possible), and the community (who best know their own preferences and needs)? How do aid workers recognize when the time is right to shift to greater community engagement and shared ownership? Better evidence, documented experience that can be shared and simple, practical advice could help answer some questions and encourage aid workers to view community engagement as a prerequisite for effective sanitation. 


\section{MONITORING \& EVALUATION OF COMMUNITY ENGAGEMENT IN SANITATION}

Systematic and documented field monitoring can be challenging in the humanitarian sector; all the more so is monitoring the effectiveness of community engagement. ALNAP's background paper on engaging communities notes that 'hard data on levels, quality and outcomes of various approaches to engaging with crisis-affected populations are scarce' (Brown et al., 2014).

In general, monitoring and evaluation (M\&E) of community engagement and sanitation differs little from standard, often participatory, approaches. Humanitarian responses that have used participatory methods to engage with people also tend to use participatory methods to monitor the results. For example, common methods mentioned include FGDs, key informant interviews, community mapping, 3-pile sorting, transect walks and observation of behaviour and of the (sanitation) environment. Projects utilizing survey approaches and Knowledge, Attitude and Practice (KAP) studies during assessments will often use the same methods to monitor the results.

The subject (the 'what') of monitoring depends on the goals of the response and the chosen activities. As described in Section 4: What is Community Engagement above, there are variations in, and some confusion about, what those goals may be, and hence what to monitor. For example, project proposals and associated logical frameworks may not clarify whether the hoped-for result of community engagement is 'instrumental' - obtaining information about community sanitation preferences and needs in order to design more appropriate facilities - or whether the process is aiming for more complex goals such as ownership and empowerment. Although often overlapping, the focus and approach to monitoring should differ according to the expected result.

In practice, monitoring may focus primarily on activities and outputs such as:

- Number of (community) trainings and meetings;

- Number and type of latrine;

- Number and type of sanitation-related non-food items (NFI) such as potties;

- Quantitative achievements in relation to standards (often Sphere) such as the ratio of latrine users to the number of latrines.

Outcomes - usually referring to behavioural change - may focus on direct or proxy indications that the affected people have accepted and owned the facilities. Such indications of success include:

- Use of the latrines by all members of the community;

- Absence of open defecation in public areas;

- The cleanliness and maintenance of the facilities;

- Levels of (representative) community engagement in the operation and maintenance of sanitation;

- Associated hygiene behaviour such as hand washing, safe disposal of children's faeces. 
The IFRC's Fast PHAST includes the following monitoring questions which provide one of the few sanitation-specific suggestions found:

- Presence and proper use of latrines;

- Absence of children's faeces in courtyard;

- Presence of bathing facilities in the household;

- Hand washing at key times (after contact with faecal matter and before handling food);

- Presence and use of dish rack;

- Presence of hand-washing facility and presence of soap or other cleaning agent.

Several of the above indicators would be monitored by staff, using internal records (e.g. for distributions) and observation (latrine use, presence and use of other facilities, counting faeces in public areas). The community itself can also be engaged with monitoring (e.g. of usage, of cleanliness), although this typically takes longer to establish; no reports were found of participatory monitoring being conducted in the first three months of an emergency.

The rarest form of monitoring (or evaluation) is at the impact level - tracking the effect of WASH on morbidity and mortality. In general, the impossibility of attribution in highly complex contexts with multiple interventions, as well as ethical concerns about control groups, makes the measuring of impact nonviable.

The quality of the latrine construction, according to some interviews, is often overlooked in monitoring, as is the quality of the community engagement process itself. Monitoring the quality of community engagement itself is more complex. Staff may record activities (such as the number of community meetings), but this does not measure their quality or whether they achieved their objectives (such as enabling representative engagement that led to changes). Stott and Keatman (2005) developed a measurement scale for community engagement as follows:

0 - no participation

1 - notified

2 - attended sessions

3 - able to express opinions (consulted)

4 - involved in the discussions (engaged)

5 - able to influence decision-making

6 - involved in decision-making ('has a vote')

7 - initiates a particular step or action point

8 - has final say/control over action to be taken

An Oxfam review in Nepal (Hawkings and O'Reilly, 2016) similarly ranked approaches in relation to an eight-point Participation Ladder ranging from passive involvement at one end of the ladder to autonomy at the other. The report comments that Oxfam 'could invest in developing a framework for participation. This would help in targeting participatory approaches and activities particularly in relation to the most vulnerable, and describing the expected outcomes of participation could lead to more focused monitoring and learning'.

Featherstone's research in Kenya and Myanmar measured the impact of three HAP accountability benchmarks (on participation, handling complaints and information sharing) through community feedback 
using an adapted Listen First methodology. Measurement of impact was participant-led, whereby, for example, participants ranked or scored their experience of the type of participation (from receiving information up to community-led decision-making) and the difference it made to the appropriateness and effectiveness of the project. The above methods were not used in the early phase of emergencies; they could be useful, but would probably need simplifying, at least initially.

It is beyond the scope of this study to suggest how community engagement can or should be monitored. Evidently there is little for practitioners to draw upon for rapid-onset emergencies and work needs to be done to create short and simple methods of measuring the extent to which communities engaged and with what effect. Proxy indicators may be the most feasible and have been used successfully, such as levels of actual use of the sanitation facilities as described above for measuring outcomes. The Core Humanitarian Standard's Guidance Notes and Indicators contains broad monitoring suggestions for key elements of community engagement. As yet though, the sector has not evidenced the central assumption - that quality community engagement will lead to more appropriate facilities that are owned and used (and possibly also lead to longer term outcomes linked to empowerment). To an extent, the evidence can be found in the sanitation interventions which failed to consult communities. These failures inadvertently provide a kind of control group for comparison with more user-led design. What is unclear is what 'good enough' community engagement actually entails in the time-poor context of a rapid-onset emergency. The systematic monitoring and evaluation of approaches and their effects is a gap waiting to be filled. 


\section{COSTS AND COST- EFFECTIVENESS}

Although there is some evidence about the cost of not engaging with communities (see the Za'atari example in Section 5: What Didn't Work and Why), agencies seem not to evaluate the cost-effectiveness of community engagement. This is perhaps not surprising, as it is potentially complex and by no means confined to this sector. The 'Cost-effectiveness of WASH interventions in emergencies was not able to be assessed. There were minimal economic outcomes or cost related data and information was inconsistent and too heterogenous for analysis' (Yates et al., 2017a). Featherstone (2013) found some evidence stating 'the literature highlighted several instances where community involvement in procurement had increased a programme's efficiency. Where communities had been empowered to monitor contractors, there was greater efficiency and value for money'. 


\section{CONCLUSIONS}

The findings suggest that the engagement of affected communities in the design and implementation of sanitation facilities is patchy and weak, with little documented evidence. There are examples of good practice, but few were reliably tested in the first three months of an emergency. Interviews tended to emphasize that the reality of community 'engagement' in the first few weeks is often little more than cursory consultation - or that it often doesn't happen at all. At the same time, interviewees were unanimous in believing that even a bare minimum of consultation could make a significant difference, and that it was feasible and could provide a basis for subsequent deeper participation and community ownership.

\subsection{SUMMARY OF APPROACHES THAT MAKE A DIFFERENCE}

- Always engage: even a few hours of rapid community engagement can make a significant difference to the appropriateness and acceptance of sanitation facilities. Rapid engagement can still ensure that women, children, the elderly, disabled and other vulnerable groups are included;

- Start with simple community engagement objectives and proposed sanitation designs;

- Create a short list of the critical information about the affected people's sanitation practices and beliefs that must be understood before facilities are designed. Again, gather feedback from vulnerable groups, women and children;

- Ensure (some) involvement of the engineers in community engagement at the outset;

- Identify effective and accepted community leaders as early as possible;

- Identify the skills available in the affected communities - don't assume outsiders are needed;

- Consult with the community about maintenance and cleaning early in the response;

- Prepare. Invest in pre-crisis skills and an understanding of the community;

- Leadership: from the WASH Cluster or government on community engagement in sanitation;

- Communicate honestly with the affected communities about the constraints and compromises imposed by the context (technical constraints, space, funding etc.);

- Assume nothing about community needs and preferences. Designs will usually need to be revisited and redesigned over time;

- Plan for a process: start simple and adapt and deepen as time and context permits. 
There needs to be a dedicated and courageous redefinition of what community engagement entails, and for what purpose, in the first few weeks of a rapid-onset emergency. Genuinely minimum standards or basic principles on engagement are needed - aiming for a sufficiency that is still effective and can be adapted to different contexts. Those who firmly believe in the virtue and efficacy of community engagement may fear that simplicity and brevity could lead to failure, or worse, do harm. To assuage those fears, these bare minimums must be designed and implemented within a framework of risk management and steady iteration - a framework that addresses the main risks of poorly implemented engagement and is designed to progressively develop approaches and levels of community engagement over time. To win the hearts and minds of those who fear that engaging with communities at all is a luxury that time and context simply do not permit - or even a potentially dangerous diversion from life-saving sanitation provision - more evidence is needed to demonstrate the efficacy (and cost savings) of engaging in ways that are practical, achievable and respectful of the affected communities' needs and current capacity.

It is not clear whether new approaches to community engagement are needed, or whether existing methods will suffice if suitably adapted to rapid-onset. If the HIF Challenge projects can capture, through well-documented monitoring and evaluation, old or new approaches to community engagement that are feasible in rapid-onset emergencies and lead to appropriate and effective sanitation, this would be a major innovation. If guidance and advice can be presented in a (very) short, simple format, that too would be an innovation. The findings would also serve as an important advocacy tool, to influence policy and address some of the structural and contextual challenges that weaken efforts to engage people in sanitation design and provision. 


\section{APPENDIX 1: LIST OF INTERVIEWEES}

- Andy Bastable, Head of Water and Sanitation, Oxfam Global Humanitarian Team

- Bibi Lamond, IRC Senior EH (WASH) Coordinator, Emergency Response Team

- Brian McSorley, Water \& Sanitation Engineering Advisor, Oxfam Global Humanitarian Team

- Frank Greaves, WASH Lead, Tearfund

- Jenny Lamb, Water \& Sanitation Engineering Advisor, Oxfam Global Humanitarian Team

- Karine Deniel, Coordinatrice de cluster EHA - Field Support Team, ACF

- Katrice King, Humanitarian WASH Advisor, Save the Children

- Libertad Gonzalez, Hygiene Promotion Advisor, Netherlands Red Cross Society

- Marion O'Reilly, Head of Public Health Promotion, Oxfam Global Humanitarian Team

- Nick Brookes, Emergency WASH Team Leader, CARE International

- Pavani Ram, Director, Community for Global Health Equity and Office of Global Health Initiatives. Associate Professor, Department of Epidemiology and Environmental Health, University of Buffalo

- Penninah Mathenge, Environment Health Sanitation and Hygiene Specialist, IRC

- Robert Fraser, Senior Officer, WASH, IFRC

- Timothy Grieve, Head of WASH, UNICEF

- William Carter, Senior Officer, WASH, IFRC 


\section{REFERENCES}

ACF (2014). Local Capacity Building Reinforcement to Increase Wash Management and Sustainability in Dare-i-Suf Bala and Payin Districts, Samangan Province, Afghanistan. Report submitted by Caterina Monti. March 2014. https://www.alnap.org/system/files/content/resource/files/main/acf-2014-afghanistan-wash\%28samangan\%29-finalevaluation.pdf

ACF (undated). Case Study: CLTS Open Defecation Free (ODF) Declaration Celebration in Tetugu. https://www.actionagainsthunger.org/sites/default/files/publications/Case Study CLTS Open Defecation Free OD F Declaration Celebration in Tetugu Otuke District Uganda 11.2011.pdf

ALNAP (2012). The State of the Humanitarian System. 2012 Edition. London: ODI, July 2012. https://www.alnap.org/system/files/content/resource/files/main/alnap-sohs-2012-lo-res.pdf

Armstrong, A. (2016). Ensuring Availability and Sustainable Management of Water and Sanitation for All. Development-orientated Relief and Recovery in the WASH Sector: A Survey of Current Practice. Briefing paper 2379 for the 39th WEDC International Conference, Kumasi, Ghana, 2016. https://wedcknowledge.lboro.ac.uk/resources/conference/39/Armstrong-2379.pdf

Bastable, A. and Russell, L. (2013). Gap Analysis in Emergency Water, Sanitation and Hygiene Promotion. HIF, July 2013. http://www.elrha.org/wp-content/uploads/2015/01/hif wash gap analysis 1.pdf

Brown, D., Donini, A. and Knox Clarke, P. (2014). Engagement of crisis-affected people in humanitarian action. Background Paper of ALNAP's 29th Annual Meeting, 11-12 March 2014, Addis Ababa. London: ALNAP/ODI. https://www.alnap.org/system/files/content/resource/files/main/background-paper-29th-meeting.pdf

Cairncross, S, Ensink, J and Kahawita, K. (2009). Evaluation of the WASH activities undertaken to prevent and control cholera outbreaks in Guinea-Conakry \& Guinea-Bissau. Systematic Literature Review. London School of Hygiene and Tropical Medicine. https://www.unicef.org/evaluation/files/LSHTM WASH Evaluation last GuineaBissau.pdf

Capps, John Meyer and Njiru, Haron (2015). 'Open Defecation Status, Community-Led Total Sanitation and Ebola Virus Disease (EVD)' in Voinjama and Kolahun Health Districts, Lofa County, Liberia (2014). Evaluation of Global Communities CLTS work funded by USAID. June 2015 https://www.globalcommunities.org/publications/GlobalCommunities-Ebola\%20Study\%20Report 081015.pdf

CARE (2015). Nepal: Rapid Gender Analysis for Sindhupalchowk. Updated 20 May 2015. https://reliefweb.int/report/nepal/nepal-rapid-gender-analysis-sindupalchowk

Dyah, Andi and Chang, Wayne (2014) unpublished. Save the Children Rapid Response: Latrines. Northwest Leyte Island, Philippines. Save the Children Methodology. Save the Children, 18 January 2014.

Featherstone, A. (2013). Improving Impact. Do Accountability Mechanisms Deliver Results? A Joint Christian Aid, Save the Children, Humanitarian Accountability Partnership Report. June 2013. https://www.alnap.org/helplibrary/improving-impact-do-accountability-mechanisms-deliver-results

Gonzalez, Libertad and Kabura, Rebecca (2006). Using PHAST for in-Country Disease Outbreaks Response. Uganda Red Cross Emergency Response During the Cholera Outbreak. June 2006. https://watsanmissionassistant.wikispaces.com/file/view/Uganda+RC+-++PHAST+in+emergency++Cholera+outbreak.pdf

Greaves, F. (2016). CLTS in Post-Emergency and Fragile States Settings, Frontiers of CLTS: Innovations and Insights 9, Brighton: IDS. http://www.communityledtotalsanitation.org/sites/communityledtotalsanitation.org/files/ Frontiers9 CLTS \%20postemergencies.pdf 
Greaves, F. (Draft 2012). Learning and recommendations on the use of CLTS in emergency and post-conflict/postemergency situations. May 2012. https://www.pseau.org/outils/ouvrages/tearfund learning and recommendations on the use of clts in emergency contexts 2012.pdf

Greaves, F. (2010). Adoption of Community-Led Total Sanitation. Guidance for programming of CLTS in Tearfundsupported projects. Tearfund. http://www.communityledtotalsanitation.org/sites/community ledtotalsanitation.org/files/media/Tearfund CLTS guidelines.pdf

Hawkings, Helen and O'Reilly, Marion. (2016) unpublished. Nepal Earthquake Wash Response. Learning Review of Community Engagement, Menstrual Hygiene Management and Capacity Building. Oxfam, March 2016.

HIF (2017). Challenge: User-centred sanitation design through rapid community engagement. Handbook 3: Call for Rapid Community Engagement Projects. HIF. ELRHA. http://www.elrha.org/hif/funding/water-sanitation-hygienewash/challenges/rapid-user-driven-sanitation-challenge/

IRIN (2013). Vandalism hampers sanitation efforts in Jordan's Za'atari camp. 19 July 2013.

http://www.irinnews.org/feature/2013/07/19/vandalism-hampers-sanitation-efforts-iordan\%E2\%80\%99sza\%E2\%80\%99atari-camp

Lloyd, A. (undated) unpublished. Methods for community engagement in sanitation for children in emergencies. Produced for Oxfam in Tanzania.

Niederberger, Eva, Ferron, Suzanne and O'Reilly, Marion (2016). Guide to community engagement in WASH. A practitioner's guide, based on lessons from Ebola. Oxfam. https://policy-practice.oxfam.org.uk/publications/quide-tocommunity-engagement-in-wash-a-practitioners-guide-based-on-lessons-fr-620139

Oxfam (undated). Urban WASH Lessons Learned from Post-Earthquake Response in Haiti. https://policypractice.oxfam.org.uk/publications/urban-wash-lessons-learned-from-post-earthquake-response-in-haiti-136538

Oxfam (2015) unpublished. Oxfam \& Partners WASH Guide to Community Mapping, Cobwebs \& Action Planning. Oxfam in Nepal, September 2015.

Oxfam (2017) unpublished. Draft Community Engagement in WASH.

Ramesh, A., Blanchet, K., Ensink, J.H.J. and Roberts, B. (2015). Evidence on the Effectiveness of Water, Sanitation, and Hygiene (WASH) Interventions on Health Outcomes in Humanitarian Crises: A Systematic Review. PLoS ONE 10(9): e0124688. doi: 10.1371/journal.pone.0124688.

http://journals.plos.org/plosone/article?id=10.1371/journal.pone.0124688

Ramos, M., Benelli, P., Irvine, E. and Watson, J. (2016). Handwashing. WASH in Emergencies. HIF Problem Exploration Report. Cardiff: ELRHA. http://www.elrha.org/wp-content/uploads/2016/01/Handwashing-WASHProblem-Exploration-Report.pdf

Sagan, Sonya and Barech, Kasim. (2011) unpublished PowerPoint. Oxfam Hyderabad. Community Led Total Sanitation (CLTS). A viable approach in emergencies? Oxfam.

Scott, R. (2013). Sustainable WASH interventions as populations transition from relief to development. Written for Tearfund UK. WEDC, February 2013. https://learn.tearfund.org/ /media/files/tilz/ topics/watsan/20130228 ofda tearfund wedc transitioning wash mainreport final.pdf?la=en

Stott, Leda and Keatman, Tracey. (2005). Tools for Exploring Community Engagement in Partnerships. Building Partnerships for Development in Water and Sanitation (BPD), May 2005.

http://www.bpdws.org/web/d/doc 267.pdf?statsHandlerDone=1

UNICEF (2015). Communication for Humanitarian Action Toolkit (CHAT). Working Version. New York: UNICEF, May 2015. http://www.unicefinemergencies.com/downloads/eresource/docs/Communication\%20for\%20Development/6C4D-CHAT Proof-2.pdf 
Wilkinson, A., Parker, M., Martineau, F. and Leach, M. (2017). Engaging 'communities': anthropological insights from the West African Ebola epidemic. Phil. Trans. R. Soc. B 372: 20160305. http://dx.doi.org/10.1098/rstb.2016.0305

WSUP (2013). Topic Brief February 2013. Getting communities engaged in water and sanitation projects: participatory design and consumer feedback. https://www.wsup.com/insights/getting-communities-engaged-in-waterand-sanitation-projects-participatory-design-and-consumer-feedbackl

Yates, T., Allen, J., Joseph, M.L. and Lantagne, D. (2017a). Short-term WASH interventions in emergency response: a systematic review. 3ie Systematic Review 33. London: International Initiative for Impact Evaluation (3ie). https://reliefweb.int/sites/reliefweb.int/files/resources/sr33-wash-interventions.pdf

Yates, T., Allen, J., Leandre Joseph, M. and Lantagne, D. (2017b). WASH interventions in disease outbreak response. Humanitarian Evidence Programme. Oxford: Oxfam GB. https://policypractice.oxfam.org.uk/publications/wash-interventions-in-disease-outbreak-response-620202 


\section{NOTES}

1. Some of the academic studies used in this review had carried out extensive literature searches in the WASH field; for example, Yates et al 2017a. reviewed 15,026 studies and evaluations, similarly evenly split between published and grey literature.

2. WHO. http://www.who.int/water sanitation health/diseases-risks/en/

3. The Grand Bargain is an agreement between more than 30 of the biggest donors and aid providers, committing them to provide 25 percent of global humanitarian funding to local and national responders by 2020 , along with more un-earmarked money, and increased multi-year funding.

https://www.agendaforhumanity.org/initiatives/3861

https://interagencystandingcommittee.org/grand-bargain-hosted-iasc 

This report was written by Peta Sandison. Oxfam acknowledges the assistance of Marion O'Reilly, Vivien Walden, Andy Bastable, and Lucy Knight in its production. It is part of a series of papers and reports written to inform public debate on development and humanitarian policy issues. The report was commissioned by the HIF/Elrha and is part of the HIF Challenge.

For further information on the issues raised in this publication please email Marion O'Reilly, Public Health Promotion Team Leader, Global Humanitarian Team (marion.o'reilly@oxfam.org).

This publication is copyright but the text may be used free of charge for the purposes of advocacy, campaigning, education, and research, provided that the source is acknowledged in full. The copyright holder requests that all such use be registered with them for impact assessment purposes. For copying in any other circumstances, or for re-use in other publications, or for translation or adaptation, permission must be secured and a fee may be charged. Email policyandpractice@oxfam.org.uk.

The information in this publication is correct at the time of going to press.

Published by Oxfam GB for Oxfam International under ISBN 978-1-78748-320-0 in August 2018

DOI: $10.21201 / 2018.3200$

Oxfam GB, Oxfam House, John Smith Drive, Cowley, Oxford, OX4 2JY, UK.

\section{OXFAM}

Oxfam is an international confederation of 20 organizations networked together in more than 90 countries, as part of a global movement for change, to build a future free from the injustice of poverty. Please write to any of the agencies for further information, or visit www.oxfam.org.

\section{HUMANITARIAN INNOVATION FUND}

Elrha's Humanitarian Innovation Fund (HIF) supports organizations and individuals to identify, nurture and share innovative and scalable solutions to the challenges facing effective humanitarian assistance. As part of this, it brings together practitioners, academics and companies in unique partnerships. 